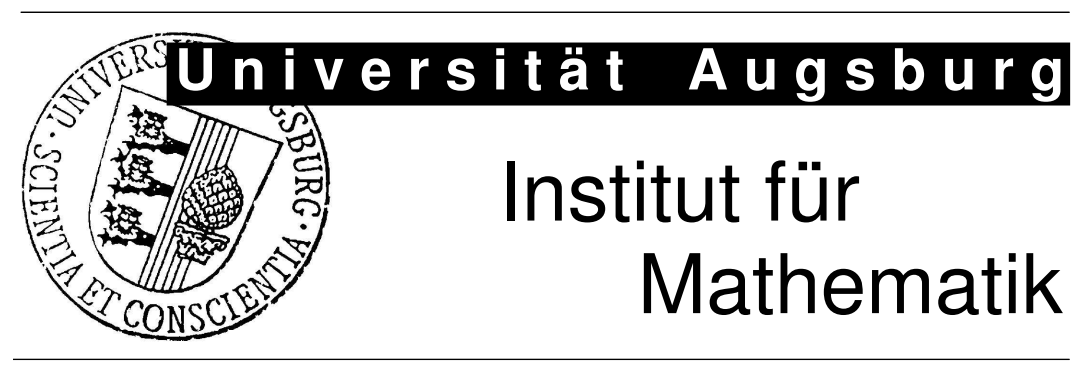

Dietrich Braess, Thomas Fraunholz, Ronald H.W. Hoppe

An Equilibrated a Posteriori Error Estimator for the Interior Penalty Discontinuous Galerkin Method 


\section{Impressum:}

\section{Herausgeber:}

Institut für Mathematik

Universität Augsburg

86135 Augsburg

http://www . math. uni-augsburg.de/de/forschung/preprints.html

\section{ViSdP:}

Ronald H.W. Hoppe

Institut für Mathematik

Universität Augsburg

86135 Augsburg

Preprint: Sämtliche Rechte verbleiben den Autoren (C) 2013 


\title{
AN EQUILIBRATED A POSTERIORI ERROR ESTIMATOR FOR THE INTERIOR PENALTY DISCONTINUOUS GALERKIN METHOD
}

\author{
D. BRAESS*, T. FRAUNHOLZ ${ }^{\dagger \S}$, AND R. H. W. HOPPE ${ }^{\dagger \ddagger}$
}

\begin{abstract}
Interior Penalty Discontinuous Galerkin (IPDG) methods for second order elliptic boundary value problems have been derived from a mixed hybrid formulation of the problem. Numerical flux functions across interelement boundaries play an important role in that theory. Residual type a posteriori error estimators for IPDG methods have been derived and analyzed by many authors including a convergence analysis of the resulting adaptive scheme $[3,12,13,14]$. Typically, the effectivity indices deteriorate with increasing polynomial order of the IPDG methods. The situation is more favorable for a posteriori error estimators derived by means of the so-called hypercircle method. Equilibrated fluxes are obtained by using an extension operator for BDM elements, and this can be done in the same way for all the DG methods presented in [2] in a unified framework. This construction enables to establish the efficiency of the equilibrated estimator, whereas the reliability can be shown by standard arguments. In contrast to the residual-type estimators, the new estimators do not contain unknown generic constants. Numerical results are given that illustrate the performance of the suggested approach.
\end{abstract}

Keywords: Interior Penalty Discontinuous Galerkin method, a posteriori error estimation, equilibration

AMS subject classification: $65 \mathrm{~N} 30,65 \mathrm{~N} 15,65 \mathrm{~N} 50$

1. Introduction. Residual a posteriori error estimates are the favorites in the error analysis of discontinuous Galerkin (DG) elements.

They are more involved than the analogous ones for conforming elements. The situation is quite different when the hypercircle method is applied.

The hypercircle method that is also denoted as two-energies principle $[4$, Section III.9] has attracted much attention, since the main contributions of the error bound do not contain (unknown) generic constants. It requires the knowledge or the construction of an equilibrated flux. This is a nontrivial task in the case of conforming Lagrangian elements. It turns out that an equilibrated flux is easily obtained from the numerical fluxes that are computed by the DG method. This holds for all the DG elements for which Arnold et al. [2] presented a unified theory. The construction of a left inverse of the divergence operator is the same for all the methods, when the mixed method in [2] is used. The numerical fluxes that are originally defined on the interelement boundaries are extended to the elements

Here, the main task is the postprocessing that yields a conforming approximation from the computed nonconforming finite element solution. In particular, the efficiency of the error bound requires a suitable procedure. Fortunately, a local construction of such an auxiliary conforming function has already been provided by the theory of

\footnotetext{
*Faculty of Mathematics, Ruhr-University, D-44780 Bochum, Germany

E-mail: Dietrich.Braess@rub.de

${ }^{\dagger}$ Inst. of Math., Univ. of Augsburg, D-86159 Augsburg, Germany

E-mail: thomas.fraunholz@math.uni-augsburg.de, hoppe@math.uni-augsburg.de

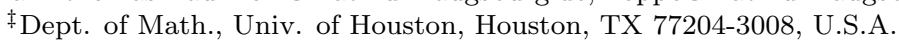

E-mail: rohop@math.uh.edu

$\S$ Supported by the DFG within the Priority Program SPP 1506.

"Supported by NSF grants DMS-1115658, DMS-1216857, by the DFG within the Priority Program SPP 1506, by the BMBF within the Collaborative Research Projects 'FROPT' and 'MeFreSim', and by the ESF within the Research Networking Programme 'OPTPDE'.
} 
residual-based estimators, and we derive efficiency of the new method by using results from the analysis of residual-based estimates [13, 14].

Our construction of equilibrated fluxes differs from the fluxes of Ern and Vohralík [10]. We use an extension operator for $B D M$ elements such that care is also taken of the curls. In this way we are able to prove efficiency of the estimates. Moreover, it becomes apparent that the construction follows the same scheme for all DG methods discussed in [2]. Only the efficiency has to be proven individually (separately) for each DG method. Here we focus on the interior penalty discontinuous Galerkin (IPDG) method.

The consideration of the efficiency shows that the estimates share a property with the estimates of other non-conforming methods [1,5]. The main contributions reflect the non-conformity, and there is no element-oriented term referring to the residue $\Delta u+f$ when we consider the problem (2.1).

Numerical experiments indicate that the quotient of the error bound and the true error do not exceed the number 2, even if the degree $k$ of the involved polynomials increases. This is consistent with results on conforming Lagrange elements. The efficiency of the error bounds by the two-energy principle is good for those elements of higher degree while the efficiency of residual estimates decrease linearly with the degree $k[6]$.

Throughout this paper we will use standard notation from Lebesgue and Sobolev space theory [4]. In particular, for a bounded domain $\Omega \subset \mathbb{R}^{2}$ we denote by $(\cdot, \cdot)_{0, \Omega}$ and $\|\cdot\|_{0, \Omega}$ the inner product and the associated norm on the Hilbert space $L^{2}(\Omega)$. We further refer to $H^{k}(\Omega), k \in \mathbb{N}$, as the Sobolev space with norm $\|\cdot\|_{k, \Omega}$ and seminorm $|\cdot|_{k, \Omega}$, whereas $H_{0}^{k}(\Omega)$ stands for the closure of $C_{0}^{\infty}(\Omega)$ with respect to the topology induced by $\|\cdot\|_{k, \Omega}$. Moreover, $H(\operatorname{div}, \Omega)$ denotes the Hilbert space of vector fields $\boldsymbol{\tau} \in L^{2}(\Omega)^{2}$ such that $\operatorname{div} \boldsymbol{\tau} \in L^{2}(\Omega)$ equipped with the graph norm.

2. Interior Penalty Discontinuous Galerkin Method. For convenience, we consider the Poisson equation

$$
\begin{array}{rll}
-\Delta u=f & \text { in } \Omega, \\
u=0 & \text { on } \Gamma,
\end{array}
$$

in a polygonal domain $\Omega \subset \mathbb{R}^{2}$ with homogeneous Dirichlet boundary conditions on $\Gamma=\partial \Omega$. The extension to more general second order elliptic differential operators and boundary conditions can be accommodated.

Let $\mathcal{T}_{h}(\Omega)$ be a simplicial triangulation of the computational domain $\Omega$. For $D \subset \bar{\Omega}$, we denote by $\mathcal{N}_{h}(D)$ and $\mathcal{E}_{h}(D)$ the set of vertices and edges of $\mathcal{T}_{h}(\Omega)$ in $D$, and we refer to $P_{k}(D), k \in \mathbb{N}$, as the set of polynomials of degree $\leq k$ on $D$. Moreover, $h_{K}, K \in \mathcal{T}_{h}(\Omega)$, and $h_{E}, E \in \mathcal{E}_{h}(\Omega)$, stand for the diameter of $K$ and the length of $E$, respectively, and $h:=\max \left(h_{K} \mid K \in \mathcal{T}_{h}(\Omega)\right)$. We consider the finite element approximation with the DG spaces

$$
\begin{aligned}
V_{h} & :=\left\{v_{h} \in L^{2}(\Omega)\left|v_{h}\right|_{K} \in P_{k}(K), K \in \mathcal{T}_{h}(\Omega)\right\}, \\
\mathbf{V}_{h} & :=\left\{\boldsymbol{\tau}_{h} \in L^{2}(\Omega)^{2}\left|\boldsymbol{\tau}_{h}\right|_{K} \in P_{k}(K)^{2}, K \in \mathcal{T}_{h}(\Omega)\right\} .
\end{aligned}
$$

For $E \in \mathcal{E}_{h}(\Omega), E=K_{+} \cap K_{-}, K_{ \pm} \in \mathcal{T}_{h}(\Omega)$, and $v_{h} \in V_{h}$, we denote the average and jump of $v_{h}$ across $E$ by $\left\{v_{h}\right\}_{E}$ and $\left[v_{h}\right]_{E}$, i.e.,

$$
\left\{v_{h}\right\}_{E}:=\frac{1}{2}\left(\left.v_{h}\right|_{E \cap T_{+}}+\left.v_{h}\right|_{E \cap T_{-}}\right), \quad\left[v_{h}\right]_{E}:=\left.v_{h}\right|_{E \cap T_{+}}-\left.v_{h}\right|_{E \cap T_{-}} .
$$


We follow the general scheme of DG methods in the mixed formulation as in [2]. The finite element approximation of the Poisson equation with homogeneous Dirichlet boundary conditions amounts to the computation of $\left(u_{h}, \boldsymbol{\sigma}_{h}\right) \in V_{h} \times \mathbf{V}_{h}$ such that for all $(v, \boldsymbol{\tau}) \in V_{h} \times \mathbf{V}_{h}$ and all $K \in \mathcal{T}_{k}$

$$
\begin{aligned}
& \int_{K} \boldsymbol{\sigma}_{h} \cdot \boldsymbol{\tau} d x=-\int_{K} u_{h} \operatorname{div} \boldsymbol{\tau} d x+\int_{\partial K} \widehat{u}_{\partial K} \boldsymbol{\nu} \cdot \boldsymbol{\tau} d s, \\
& \int_{K} \boldsymbol{\sigma}_{h} \cdot \operatorname{grad} v d x=\int_{K} f v d x+\int_{\partial K} \widehat{\boldsymbol{\sigma}}_{\partial K} \cdot \boldsymbol{\nu} v d s,
\end{aligned}
$$

where $\boldsymbol{\nu}$ stands for the exterior normal unit vector on $\partial K$.

The definition of the DG method is completed by fixing $\widehat{u}_{\partial K}$ and the numerical fluxes $\widehat{\boldsymbol{\sigma}}_{\partial K}$. In particular, the IPDG method is obtained by the specification of the numerical fluxes $\widehat{u}_{\partial K}$ and $\widehat{\boldsymbol{\sigma}}_{\partial K}$ :

$$
\left.\begin{array}{rl}
\left.\widehat{u}_{\partial K}\right|_{E} & :=\left\{u_{h}\right\}_{E}, \\
\left.\widehat{\boldsymbol{\sigma}}_{\partial K}\right|_{E} & :=\left\{\operatorname{grad} u_{h}\right\}_{E}-\alpha h_{E}^{-1}\left[u_{h}\right]_{E} \boldsymbol{\nu},
\end{array}\right\} E \in \mathcal{E}_{h}(\Omega),
$$

where $\alpha>0$ is a penalty parameter, and $\alpha=2.5(k+1)^{2}$ is considered as a convenient choice [11].

3. An Interpolation by BDM Elements. The numerical fluxes $\widehat{\sigma}$ that live on the interelement boundaries will be extended to the elements by an interpolation. The finite element space for the fluxes is the $\mathrm{BDM}$ element, where $\mathbf{B D M}_{k}(K), k \in \mathbb{N}$, is given by

$$
\mathbf{B D M}_{k}(K)=P_{k}(K)^{2}, \quad \operatorname{dim} \mathbf{B D M}_{k}(K)=(k+1)(k+2) .
$$

We refer to $\lambda_{i}^{K}, 1 \leq i \leq 3$, as the barycentric coordinates of $K \in \mathcal{T}_{h}(\Omega)$ and denote by $b_{K}$ the element bubble function $b_{K}:=\lambda_{1}^{K} \lambda_{2}^{K} \lambda_{3}^{K}$. By $(3.41)$ in [2, p. 125] any $\mathbf{q}_{K} \in \mathbf{B D M}_{k}(K)$ is uniquely determined by the following degrees of freedom (DOF)

$$
\begin{aligned}
& \int_{E} \mathbf{q}_{K} \cdot \boldsymbol{\nu} p_{k} d s, \quad p_{k} \in P_{k}(E), E \in \mathcal{E}_{h}(\partial K), \\
& \int_{K} \mathbf{q}_{K} \cdot \operatorname{grad} p_{k-1} d x, \quad p_{k-1} \in P_{k-1}(K), \\
& \int_{K} \mathbf{q}_{K} \cdot \operatorname{curl}\left(b_{K} p_{k-2}\right) d x, \quad p_{k-2} \in P_{k-2}(K) .
\end{aligned}
$$

A standard scaling argument yields a bound of the $L^{2}$ norm when a BDM element is interpolated with these data.

LEMMA 3.1. There exists a constant $c$ that depends only on $k$ and the shape regularity of $\mathcal{T}_{h}$ such that for each $\mathbf{q}_{K} \in \mathbf{B D M}_{k}(K)$ :

$$
\begin{aligned}
\int_{K} \mathbf{q}_{K}^{2}(x) d x \leq & \left(h \int_{\partial K}\left(\mathbf{q}_{K} \cdot \boldsymbol{\nu}\right)^{2} d s\right. \\
& +h^{2} \max \left\{\int_{K}\left(\mathbf{q}_{K} \operatorname{grad} p\right)^{2} d x ; p \in P_{k-1}, \max _{x \in K}|p(x)| \leq 1\right\} \\
& \left.+h^{2} \max \left\{\int_{K}\left(\mathbf{q}_{K} \cdot \operatorname{curl}\left(b_{K} p\right)\right)^{2} d x ; p \in P_{k-2}, \max _{x \in K}|p(x)| \leq 1\right\}\right) .
\end{aligned}
$$



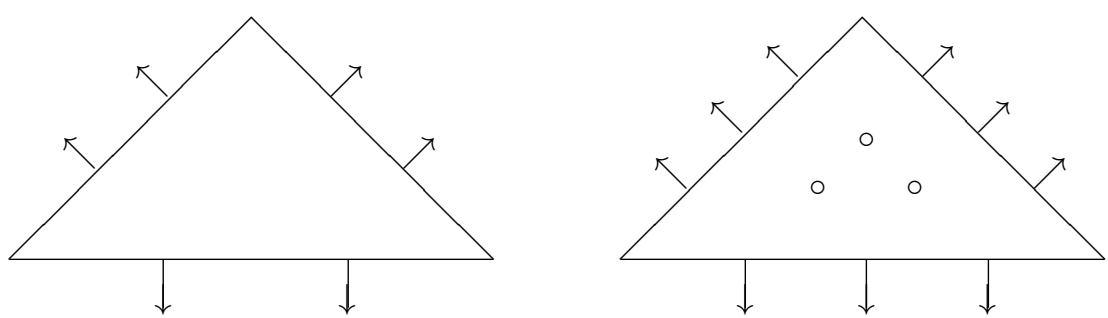

FIG. 3.1. DOFs of the BDM 1 element and of the BDM $M_{2}$ element.

Remark 3.2. For $k=1, \mathbf{q}_{K} \in \mathbf{B D M}_{1}(K)$ is uniquely determined by the DOF on $\partial K$; cf. Figure 3.1 .

REMARK 3.3. A BDM element may be specified by $\operatorname{div} \mathbf{q}_{K}$ instead of (3.2b). Therefore, the bound in Lemma 3.1 can be replaced by

$$
\begin{aligned}
\int_{K} \mathbf{q}_{K}^{2}(x) d x & \leq c\left(h \int_{\partial K}\left(\mathbf{q}_{K} \cdot \boldsymbol{\nu}\right)^{2} d s+h^{2} \int_{K}\left(\operatorname{div} \mathbf{q}_{K}\right)^{2} d x\right. \\
& \left.+h^{2} \max \left\{\int_{K}\left(\mathbf{q}_{K} \cdot \operatorname{curl}\left(b_{K} p\right)\right)^{2} d x ; p \in P_{k-2}, \max _{x \in K}|p(x)| \leq 1\right\}\right) .
\end{aligned}
$$

Moreover, we will refer to the following

Lemma 3.4. There exists a constant $c$ that depends only on $k$ and the shape regularity of $\mathcal{T}_{h}$ such that for each $\mathbf{q}_{K} \in \mathbf{B D M}_{k}(K)$ :

$$
\left\|\mathbf{q}_{K} \cdot \boldsymbol{\nu}\right\|_{0, \partial K} \leq c h^{-1 / 2}\left\|\mathbf{q}_{K}\right\|_{0, K} .
$$

This inequality follows from the fact that

$$
\inf _{\left\|\mathbf{q}_{K} \cdot \nu\right\|_{0, \partial K}=1}\left\|\mathbf{q}_{K}\right\|_{0, K}>0 .
$$

The constant $c$ depends on the degree $k$, since (3.3) is not true, if we take the infimum over all $H^{1}$ functions.

4. Application of the hypercircle method to nonconforming finite elements. The starting point is the Theorem of Prager and Synge $[4,15]$ that is also called the two-energies principle. We restrict ourselves to the Poisson equation; the generalization to other elliptic problems can be found in [4, Ch. III, §9].

Theorem 4.1. (Theorem of Prager and Synge, Two-Energies principle). Let $\boldsymbol{\sigma} \in H(\operatorname{div}, \Omega)$ and $v \in H_{0}^{1}(\Omega)$. Furthermore, let $u$ be the solution of (2.1). If $\boldsymbol{\sigma}$ satisfies the equilibrium condition

$$
\operatorname{div} \boldsymbol{\sigma}+f=0,
$$

then,

$$
|u-v|_{1, \Omega}^{2}+\|\operatorname{grad} u-\boldsymbol{\sigma}\|_{0, \Omega}^{2}=\|\operatorname{grad} v-\boldsymbol{\sigma}\|_{0, \Omega}^{2} .
$$

Supplement. Let $J(v):=\frac{1}{2}\|\operatorname{grad} v\|_{0, \Omega}^{2}-\int_{\Omega} f v d x$ and $J^{c}(\boldsymbol{\sigma}):=\frac{1}{2}\|\boldsymbol{\sigma}\|_{0, \Omega}^{2}$ denote the (direct) energy and the complementary energy, respectively. If the assumptions above hold, then

$$
|u-v|_{1, \Omega}^{2}+\|\operatorname{grad} u-\boldsymbol{\sigma}\|_{0, \Omega}^{2}=2 J(v)+2 J^{c}(\boldsymbol{\sigma}) .
$$


A proof is provided, e.g., in [4]

The piecewise gradient of a finite element function $v_{h}$ in the broken $H^{1}$ space will be denoted by $\operatorname{grad}_{\mathrm{h}} v_{h}$. We have $\operatorname{grad}_{\mathrm{h}} v_{h} \in L^{2}(\Omega)$.

COROLLARY 4.2. Let $u_{h}$ be the finite element solution of a nonconforming method in a broken $H^{1}$ space, e.g., a DG method. Assume that an auxiliary function $u_{h}^{\text {conf }} \in$ $H^{1}(\Omega)$ that satisfies the Dirichlet boundary condition, is obtained by postprocessing. Moreover, let $\boldsymbol{\sigma}_{h}^{\mathrm{eq}} \in H(\operatorname{div}, \Omega)$ be a flow that satisfies the equilibrium condition (4.1). Then we have the estimate

$$
\left\|\operatorname{grad} u-\operatorname{grad}_{\mathrm{h}} u_{h}\right\|_{0, \Omega} \leq\left\|\operatorname{grad}_{\mathrm{h}} u_{h}-\sigma_{h}^{\text {eq }}\right\|_{0, \Omega}+2\left\|\operatorname{grad}_{\mathrm{h}} u_{h}-\operatorname{grad} u_{h}^{\text {conf }}\right\|_{0, \Omega} .
$$

Indeed, the two-energies principle yields the following bound for the auxiliary function $u_{h}^{\text {conf }}$ :

$$
\left\|\operatorname{grad} u-\operatorname{grad} u_{h}^{\mathrm{conf}}\right\|_{0, \Omega} \leq\left\|\operatorname{grad} u_{h}^{\mathrm{conf}}-\boldsymbol{\sigma}_{h}^{\mathrm{eq}}\right\|_{0, \Omega} .
$$

By applying the triangle inequality twice, we obtain (4.2).

The corollary was implicitly used in $[1,5]$.

In actual computations, we have frequently an additional term due to data oscillations. We only have the equilibration for an approximate function $\bar{f}$, i.e.,

$$
\operatorname{div} \sigma+\bar{f}=0 .
$$

If $f_{h}$ is the best approximation of $f$ by a piecewise polynomial of degree $k-1$, then an extra term

$$
\operatorname{ch}\|f-\bar{f}\|_{0, \Omega}
$$

has to be added to the error bound. Since the term is considered as a term of higher order, it is no drawback to have here a generic constant $c$. As usual, there is a generic constant only in this contribution to the a posteriori error bound.

5. Equilibration. Let $\bar{f}$ be the $L^{2}$-projection of $f$ onto piecewise polynomials of degree $k-1$, i.e.,

$$
\int_{K} \bar{f} v d x=\int_{K} f v d x, \quad v \in P_{k-1}(K) .
$$

We construct a flux $\widehat{\boldsymbol{\sigma}}_{K} \in \mathbf{B D M}_{k}(K)$ by the specifications

$$
\begin{aligned}
\left.\widehat{\boldsymbol{\sigma}}_{K}\right|_{\partial K} & =\widehat{\boldsymbol{\sigma}}_{\partial K}, \\
\int_{K} \widehat{\boldsymbol{\sigma}}_{K} \cdot \operatorname{grad} p_{k-1} d x & =\int_{K} \boldsymbol{\sigma}_{h} \cdot \operatorname{grad} p_{k-1} d x, \quad p_{k-1} \in P_{k-1}(K), \\
\int_{K} \widehat{\boldsymbol{\sigma}}_{K} \cdot \operatorname{curl}\left(b_{K} p_{k-2}\right) d x & =\int_{K} \boldsymbol{\sigma}_{h} \cdot \operatorname{curl}\left(b_{K} p_{k-2}\right) d x, \quad p_{k-2} \in P_{k-2}(K) .
\end{aligned}
$$

The first equation corresponds to (3.2a) and shows that the flux is an extension of the numerical flux that is originally defined on the element boundaries. Now, it follows 
from (5.2a), Gauss' theorem, (5.1), and the DG finite element equation (2.3b) that

$$
\begin{aligned}
\int_{K} \operatorname{div} \widehat{\boldsymbol{\sigma}}_{K} p_{k-1} d x & =-\int_{K} \widehat{\boldsymbol{\sigma}}_{K} \cdot \operatorname{grad} p_{k-1} d x+\int_{\partial K} \widehat{\boldsymbol{\sigma}}_{K} \cdot \boldsymbol{\nu}_{K} p_{k-1} d x \\
& =-\int_{K} \boldsymbol{\sigma}_{K} \cdot \operatorname{grad} p_{k-1} d x+\int_{\partial K} \widehat{\boldsymbol{\sigma}}_{K} \cdot \boldsymbol{\nu}_{K} p_{k-1} d x \\
& =-\int_{K} f p_{k-1} d x=-\int_{K} \bar{f} p_{k-1} d x
\end{aligned}
$$

Since $\operatorname{div} \widehat{\boldsymbol{\sigma}}_{K}$ and $\bar{f}$ are contained in $P_{k-1}(K)$, we readily deduce from (5.3) that

$$
\operatorname{div} \widehat{\boldsymbol{\sigma}}_{K}+\bar{f}=0 \text {. }
$$

Therefore, we have obtained an equilibrated flux up to data oscillations.

The last specification (5.2c) aims at the minimization of the error bound with respect to the known quantities. This is one difference to the equilibration procedure by Ern and Vohralík [10] who used Raviart-Thomas elements.

REMARK 5.1. If $k=1$, due to Remark 3.2, $\widehat{\boldsymbol{\sigma}}_{K}$ is uniquely defined by (5.2a), that is by data of the numerical flux on the edges.

Note that up to now we have not used the specification (2.4) of the interior penalty method IPDG.

6. An approximation by conforming elements. Corollary 4.2 shows that we require an approximation of $u_{h}$ by an $H^{1}$ function. We want to have a conforming element $u_{h}^{\text {conf }}$, and we will compute the norm $\left\|\operatorname{grad} u_{h}^{\text {conf }}-\operatorname{grad}_{\mathrm{h}} u_{h}\right\|_{0, \Omega}$. If we are content with an estimate of this quantity, then an unknown generic constant as in (6.2) would enter into the a posteriori error estimate. Since such an approximation was also used in [13] in connection with residual-based error estimates, we recall the construction in [13].

Let $\mathcal{N}^{L}$ be the set of Lagrangian nodal points for the elements in $V_{h}^{r}$. Let $\kappa_{i}$ be the number of triangles that share the nodal point $x_{i} \in \mathcal{N}^{L}$. We have $\kappa_{i}=1$, if $x_{i}$ is contained in the interior of an element, while $\kappa_{i}>1$, if $x_{i} \in \mathcal{N}^{L} \cap \mathcal{E}_{h}(\Omega)$. The multiplicity $\kappa_{i}$ is bounded, since a minimal angle condition is assumed. The associated conforming element is now defined by its nodal values

$$
u_{h}^{\text {conf }}\left(x_{i}\right):=\left.\frac{1}{\kappa_{i}} \sum_{K \in \mathcal{T}_{h}, x_{i} \in K} u_{h}\right|_{K}\left(x_{i}\right) .
$$

The following estimate is provided by Theorem 2.2 in [13]:

$$
\sum_{K \in \mathcal{T}_{h}}\left\|\operatorname{grad} u_{h}^{\text {conf }}-\operatorname{grad}_{\mathrm{h}} u_{h}\right\|_{0, K}^{2} \leq c \sum_{E \in \mathcal{E}_{h}} h^{-1} \|\left.\left[u_{h}\right]\right|_{0, E} ^{2} \cdot
$$

The constant $c$ depends only on the degree $k$ of the finite elements and the shape regularity of the triangulation. Note that the quasi-local character is more apparent in the formulation

$$
\left\|\operatorname{grad} u_{h}^{\text {conf }}-\operatorname{grad}_{\mathrm{h}} u_{h}\right\|_{0, K}^{2} \leq c \sum_{E \in \mathcal{E}_{h}, E \cap K \neq \emptyset} h^{-1} \|\left.\left[u_{h}\right]\right|_{0, E} ^{2} .
$$

The right-hand side of (6.2), in turn, can be bounded by Theorem 3.2(iv) in [14]:

$$
\sum_{E \in \mathcal{E}_{h}} h^{-1}\left\|\left.\left[u_{h}\right]\right|_{0, E} ^{2} \leq c\right\| \operatorname{grad} u-\operatorname{grad}_{\mathrm{h}} u_{h} \|_{0, \Omega}^{2},
$$


and we eventually obtain

$$
\left\|\operatorname{grad} u_{h}^{\text {conf }}-\operatorname{grad}_{\mathrm{h}} u_{h}\right\|_{0, \Omega}^{2} \leq c\left\|\operatorname{grad} u-\operatorname{grad}_{\mathrm{h}} u_{h}\right\|_{0, \Omega}^{2} .
$$

This inequality will be used for the verification of the efficiency of the a posteriori error bound under consideration.

The inequality (6.4) is obtained from the efficiency of a residual a posteriori error estimate [13]. It gives rise to a comparison theorem in the spirit of the results in [5].

TheOREM 6.1. Let $u_{h}^{G}$ be the solution of the Poisson equation by the conforming finite elements $V_{h} \cap H^{1}(\Omega)$ on the same triangulation. Then

$$
\left\|\operatorname{grad}\left(u-u_{h}^{G}\right)\right\|_{0, \Omega} \leq c\left\|\operatorname{grad}_{\mathrm{h}}\left(u-u_{h}\right)\right\|_{0, \Omega} .
$$

Proof. From the Galerkin orthogonality $\left(\operatorname{grad}\left(u-u_{h}^{G}\right), \operatorname{grad} v\right)_{0, \Omega}=0$ for all $v \in V_{h} \cap H^{1}(\Omega)$ it follows that $\left\|\operatorname{grad}\left(u-u_{h}^{G}\right)\right\|_{0, \Omega} \leq\left\|\operatorname{grad}\left(u-u_{h}^{\text {conf }}\right)\right\|_{0, \Omega}$. Now we obtain from $(6.4)$

$$
\begin{aligned}
\left\|\operatorname{grad}\left(u-u_{h}^{G}\right)\right\|_{0, \Omega} & \leq\left\|\operatorname{grad}\left(u-u_{h}^{\mathrm{conf}}\right)\right\|_{0, \Omega} \\
& \leq\left\|\operatorname{grad}_{h}\left(u-u_{h}\right)\right\|_{0, \Omega}+\left\|\operatorname{grad}_{h}\left(u_{h}-u_{h}^{\mathrm{conf}}\right)\right\|_{0, \Omega} \\
& \leq\left\|\operatorname{grad}_{h}\left(u-u_{h}\right)\right\|_{0, \Omega}+c\left\|\operatorname{grad}_{h}\left(u-u_{h}\right)\right\|_{0, \Omega},
\end{aligned}
$$

and the proof is complete.

We note that the comparison theorem was established independently of the equilibration, and the data oscillation is not involved.

7. Efficiency. Let $\widehat{\boldsymbol{\sigma}}_{h} \in H(\operatorname{div}, \Omega)$ with $\left.\widehat{\boldsymbol{\sigma}}_{h}\right|_{K} \in \mathbf{B D M}_{k}(K), K \in \mathcal{T}_{h}(\Omega)$, be the equilibrated flux constructed according to (5.2) and let $u_{h}^{\text {conf }} \in V_{h} \cap H^{1}(\Omega)$ be defined by the averaging procedure from the previous section. Recalling Corollary 4.2 we introduce the estimator

$$
\begin{aligned}
& \eta_{\text {hyp }}:=\eta_{\text {hyp }}^{(1)}+\eta_{\text {hyp }}^{(2)}, \quad \eta_{\text {hyp }}^{(\nu)}:=\sum_{K \in \mathcal{T}_{h}(\Omega)} \eta_{K}^{(\nu)}, \quad 1 \leq \nu \leq 2, \\
& \eta_{K}^{(1)}:=\left\|\operatorname{grad}_{\mathrm{h}} u_{h}-\widehat{\boldsymbol{\sigma}}_{h}\right\|_{0, K}, \quad \eta_{K}^{(2)}:=2\left\|\operatorname{grad}_{\mathrm{h}} u_{h}-\operatorname{grad} u_{h}^{\operatorname{conf}}\right\|_{0, K}, \quad K \in \mathcal{T}_{h}(\Omega) .
\end{aligned}
$$

By Corollary 4.2 we get the reliable a posteriori error estimate

$$
\left\|\operatorname{grad} u-\operatorname{grad}_{\mathrm{h}} u_{h}\right\|_{0, h} \leq \eta_{\text {hyp }}+c h\left\|f-f_{h}\right\|_{0, \Omega}
$$

with a generic constant only in the term with the data oscillation. From (6.4) it follows that the efficiency of the error bound (7.2) without the contribution of the data oscillation is guaranteed when we have appropriate bounds for $\left\|\operatorname{grad}_{\mathrm{h}} u_{h}-\widehat{\boldsymbol{\sigma}}_{h}\right\|_{0, \Omega}$. To this end, we will establish bounds for the terms in the triangle inequality $\| \operatorname{grad}_{\mathrm{h}} u_{h}-$ $\widehat{\boldsymbol{\sigma}}_{h}\left\|_{0, \Omega} \leq\right\| \operatorname{grad}_{\mathrm{h}} u_{h}-\boldsymbol{\sigma}_{h}\left\|_{0, \Omega}+\right\| \boldsymbol{\sigma}_{h}-\widehat{\boldsymbol{\sigma}}_{h} \|_{0, \Omega}$.

First, (2.3a) and Gauss' theorem yield for $\boldsymbol{\tau} \in \mathbf{V}_{h}$ :

$$
\begin{aligned}
\int_{K}\left(\boldsymbol{\sigma}_{h}-\operatorname{grad}_{\mathrm{h}} u_{h}\right) \cdot \boldsymbol{\tau} d x= & \int_{K} \boldsymbol{\sigma}_{h} \cdot \boldsymbol{\tau} d x-\int_{K} \operatorname{grad}_{\mathrm{h}} u_{h} \cdot \boldsymbol{\tau} d x \\
= & -\int_{K} u_{h} \operatorname{div} \boldsymbol{\tau} d x+\int_{\partial K} \hat{u} \boldsymbol{\nu} \boldsymbol{\tau} d x \\
& +\int_{K} u_{h} \operatorname{div} \boldsymbol{\tau} d x-\int_{\partial K} u_{h} \boldsymbol{\nu} \cdot \boldsymbol{\tau} d x \\
= & \int_{\partial K}\left(\hat{u}-u_{h}\right) \boldsymbol{\nu} \cdot \boldsymbol{\tau} d x .
\end{aligned}
$$


It follows from the specification of the internal penalty method (2.4) that $\hat{u}_{K}-u_{h}=$ $\frac{1}{2}\left[u_{h}\right]_{E}$ holds on $E \subset \partial K$. We set $\boldsymbol{\tau}:=\boldsymbol{\sigma}_{h}-\operatorname{grad}_{\mathrm{h}} u_{h}$, and a standard scaling argument yields

$$
\left\|\boldsymbol{\sigma}_{h}-\operatorname{grad}_{\mathrm{h}} u_{h}\right\|_{0, K} \leq c h^{-1 / 2}\left\|\left[u_{h}\right]\right\|_{0, \partial K}
$$

After summing over all elements we obtain with (6.3) the required bound for the left-hand side of (7.3),

$$
\left\|\boldsymbol{\sigma}_{h}-\operatorname{grad}_{\mathrm{h}} u_{h}\right\|_{0, \Omega} \leq c\left\|\operatorname{grad} u-\operatorname{grad}_{\mathrm{h}} u_{h}\right\|_{0, \Omega} .
$$

Moreover, it follows from Lemma 3.4 and (7.3) that

$$
\left\|\left(\boldsymbol{\sigma}_{h}-\operatorname{grad}_{\mathrm{h}} u_{h}\right) \cdot \boldsymbol{\nu}\right\|_{0, \partial K} \leq c h^{-1 / 2}\left\|\left[u_{h}\right]\right\|_{0, \partial K} .
$$

Eventually we derive a bound for $\widehat{\boldsymbol{\sigma}}_{h}-\boldsymbol{\sigma}_{h}$. Lemma 3.1 together with $(5.2 \mathrm{~b})$ and (5.2c) yields

$$
\left\|\widehat{\boldsymbol{\sigma}}_{h}-\boldsymbol{\sigma}_{h}\right\|_{0, K} \leq c h^{1 / 2}\left\|\left(\widehat{\boldsymbol{\sigma}}_{h}-\boldsymbol{\sigma}_{h}\right) \cdot \boldsymbol{\nu}\right\|_{0, \partial K} \cdot
$$

Recalling the specification (2.4) for the IPDG method, we obtain on $E \subset \partial K$

$$
\begin{aligned}
\widehat{\boldsymbol{\sigma}}_{h}-\boldsymbol{\sigma}_{h} & =\widehat{\boldsymbol{\sigma}}_{h}-\operatorname{grad}_{\mathrm{h}} u_{h}+\left(\operatorname{grad}_{\mathrm{h}} u_{h}-\boldsymbol{\sigma}_{h}\right) \\
& =\frac{1}{2}\left[\operatorname{grad}_{\mathrm{h}} u_{h}\right]-\alpha h_{E}^{-1}\left[u_{h}\right] \boldsymbol{\nu}+\left(\operatorname{grad}_{\mathrm{h}} u_{h}-\boldsymbol{\sigma}_{h}\right) .
\end{aligned}
$$

Let $E=\partial K \cap \partial K^{\prime}$. Lemma 3.2(ii) in [14] asserts that

$$
\left\|\left[\operatorname{grad}_{\mathrm{h}} u_{h}\right] \cdot \boldsymbol{\nu}\right\|_{0, E} \leq c h^{-1 / 2}\left\|\operatorname{grad} u-\operatorname{grad}_{\mathrm{h}} u_{h}\right\|_{0, K \cup K^{\prime}} .
$$

The second term in (7.5) is already estimated in (6.3). The third term is reduced by (7.4) also to the second one, and we get

$$
\sum_{K \in \mathcal{T}_{h}}\left\|\widehat{\boldsymbol{\sigma}}_{h}-\boldsymbol{\sigma}_{h}\right\|_{0, K}^{2} \leq c \sum_{K \in \mathcal{T}_{h}}\left\|\operatorname{grad} u-\operatorname{grad}_{\mathrm{h}} u_{h}\right\|_{0, K}^{2} .
$$

By collecting all terms we obtain the efficiency of the a posteriori error estimate deduced from Corollary 4.2 .

THEOREM 7.1. Let $u_{h}$ and $\widehat{\boldsymbol{\sigma}}_{h}$ be the finite element solution of the IPDG method and the equilibrated flux, respectively. Further, assume that a conforming function $u_{h}^{\text {conf }}$ has been constructed as described in Section 6 . There is a constant c that depends only on the degree $k$ and the shape regularity of the triangulation such that

$$
\eta_{\text {hyp }} \leq c\left\|\operatorname{grad} u-\operatorname{grad}_{\mathrm{h}} u_{h}\right\|_{0, \Omega} \text {. }
$$

8. Numerical results. In this section, we present a documentation of numerical results for two representative examples illustrating the performance of the suggested adaptive approach which consists of successive cycles of the steps

$$
\text { SOLVE } \Longrightarrow \text { ESTIMATE } \Longrightarrow \text { MARK } \Longrightarrow \text { REFINE. }
$$

In the step SOLVE we compute the solution of the IPDG approximation (2.3), whereas the second step ESTIMATE is devoted to the computation of the local components 
$\eta_{K}^{(1)}$ and $\eta_{K}^{(2)}$ of the error estimator $\eta_{\text {hyp }}$ (cf. (7.1)). We use the standard Dörfler marking in step MARK: Given some bulk parameter $0<\theta \leq 1$, we choose a set $\mathcal{M} \subseteq \mathcal{T}_{h}(\Omega)$ of elements $K \in \mathcal{T}_{h}(\Omega)$ such that

$$
\theta \eta_{\text {hyp }} \leq \sum_{K \in \mathcal{M}}\left(\eta_{K}^{(1)}+\eta_{K}^{(2)}\right) .
$$

The final step REFINE takes care of the practical realization of the refinement process of the elements in $\mathcal{M}$ and is based on newest vertex bisection [9].

Example 1: We consider the Laplace equation with inhomogeneous Dirichlet boundary conditions

$$
\begin{aligned}
-\Delta u=0 & \text { in } \Omega, \\
u=g & \text { on } \partial \Omega,
\end{aligned}
$$

in the L-shaped domain $\Omega:=(-1,+1)^{2} \backslash[0,+1) \cup(-1,0]$, where $g$ in $(8.2 \mathrm{~b})$ is chosen such that

$$
u(r, \varphi)=r^{2 / 3} \sin (2 \varphi / 3)
$$

is the exact solution (in polar coordinates). The solution exhibits a singularity at the origin.
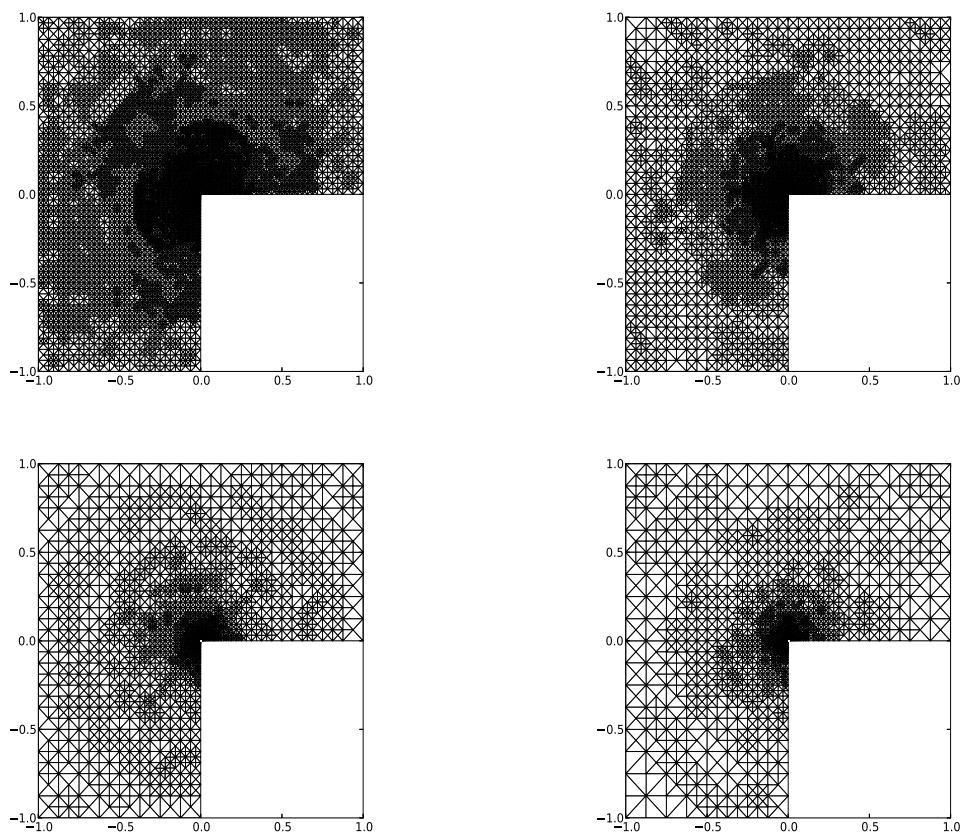

FIG. 8.1. Example 1: Adaptively refined meshes for $k=1$ (top left), $k=2$ (top right), $k=3$ (bottom left), and $k=4$ (bottom right) after 9 adaptive cycles $(\theta=0.3$ in the Dörfler marking).

For $\theta=0.3$ in the Dörfler marking, Figure 8.1 displays the adaptively refined meshes for polynomial degrees $1 \leq k \leq 4$. As can be expected, the adaptive algorithm refines in the vicinity of the origin with coarser meshes for increasing polynomial degree $k$. 

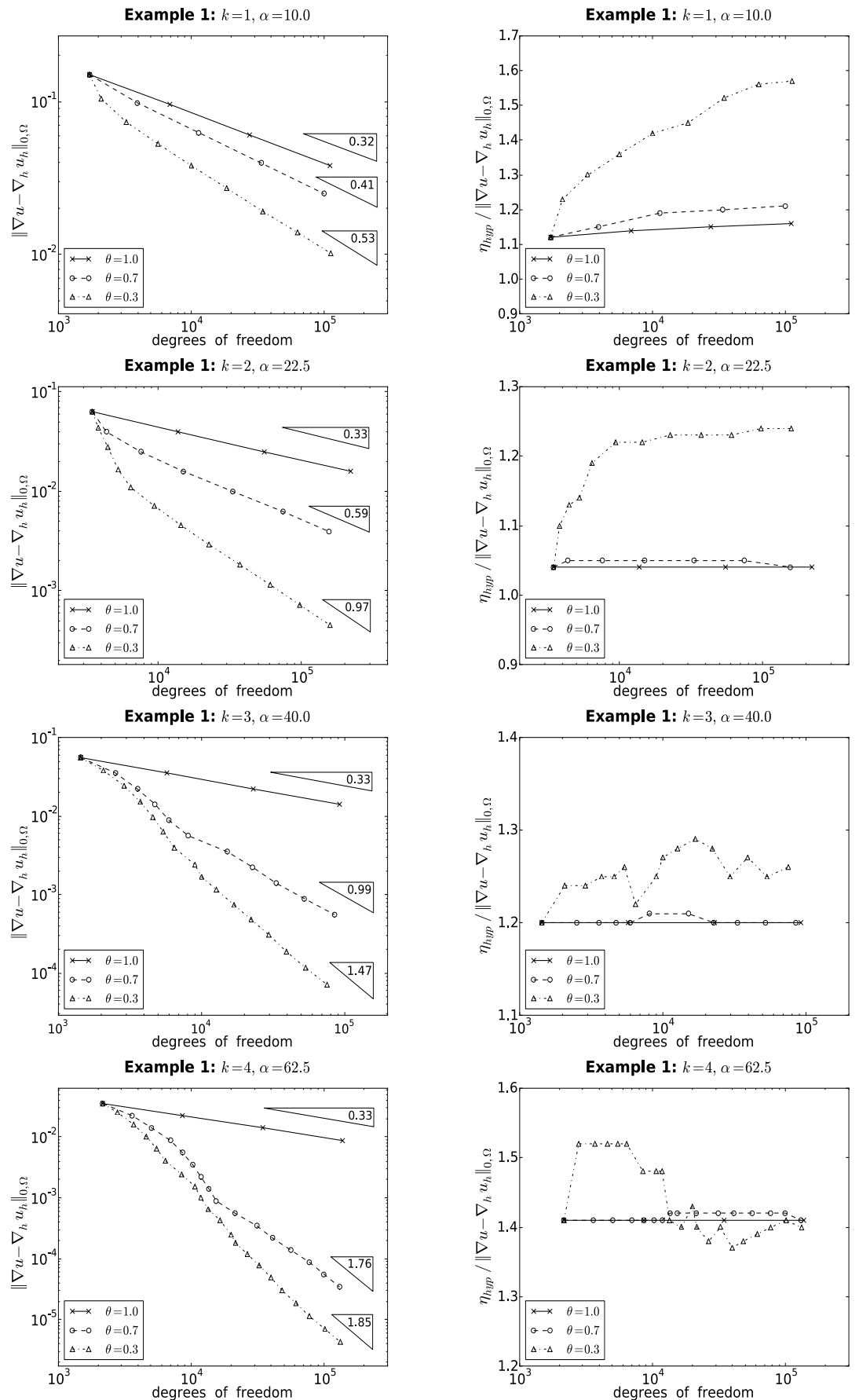

FIG. 8.2. Example 1: The discretization error $\left\|\operatorname{grad} u-\operatorname{grad}_{\mathrm{h}} u_{h}\right\|_{0, \Omega}$ as a function of the degrees of freedom (DOF) on a logarithmic scale for various $\theta$ in the Dörfler marking (left) and the associated effectivity indices (right), i.e., the quotients of the estimated and the exact error. 
For adaptive refinements with $\theta=0.7, \theta=0.3$, and also for $\theta=1$ (uniform refinement), Figure 8.2 (left) shows the decrease of the global discretization error $\left\|\operatorname{grad} u-\operatorname{grad}_{\mathrm{h}} u_{h}\right\|_{0, \Omega}$ as a function of the total number of degrees of freedom (DOF) on a logarithmic scale for polynomial degree $k=1$ (top left) to $k=4$ (bottom left). The negative slope is indicated for each curve.

We see that the optimal convergence rates are approached asymptotically for $\theta=0.3$. Figure (8.2) (right) displays the associated effectivity indices (ratio of the a posteriori error estimator and the global discretization error). In contrast to standard residual type a posteriori error estimators for IPDG approximations, the effectivity indices are only slightly above 1 , remain below 1.6 , and do not significantly deteriorate with increasing polynomial degree $k$.

Example 2: We consider Poisson's equation with homogeneous Dirichlet boundary conditions

$$
\begin{aligned}
-\Delta u=f & \text { in } \Omega, \\
u=0 & \text { on } \partial \Omega,
\end{aligned}
$$

in the unit square $\Omega=(0,1)^{2}$, where the right-hand side $f$ in $(8.3 \mathrm{a})$ is chosen such that

$$
u(x, y)=x(1-x) y(1-y) \arctan (60(r-1)), r^{2}:=(x-5 / 4)^{2}+(y+1 / 4)^{2}
$$

is the exact solution. The solution exhibits an interior layer along a circular segment inside the computational domain.
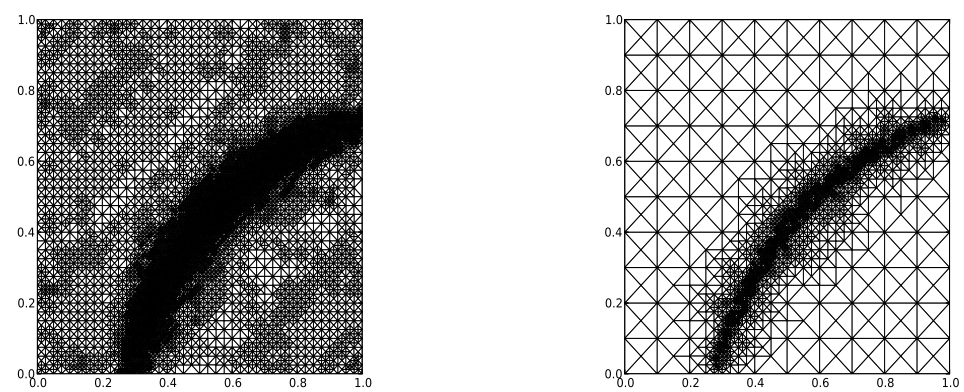

FIG. 8.3. Example 2: Adaptively refined meshes for $k=1$ (left) and $k=4$ (right) after 9 adaptive cycles $(\theta=0.3$ in the Dörfler marking).

Figure 8.3 shows the adaptively refined meshes for polynomial degree $k=1$ and $k=4$ in case of $\theta=0.3$ in the Dörfler marking, whereas for uniform refinement $(\theta=1), \theta=0.7$, and $\theta=0.3$ Figure 8.4 displays the global discretization error as a function of the DOF on a logarithmic scale (left) and the associated effectivity indices (right). We see that both for $\theta=0.7$ and $\theta=0.3$ the optimal convergence rates are achieved asymptotically and that the effectivity indices are even slightly improved with increasing polynomial degree $k$. 

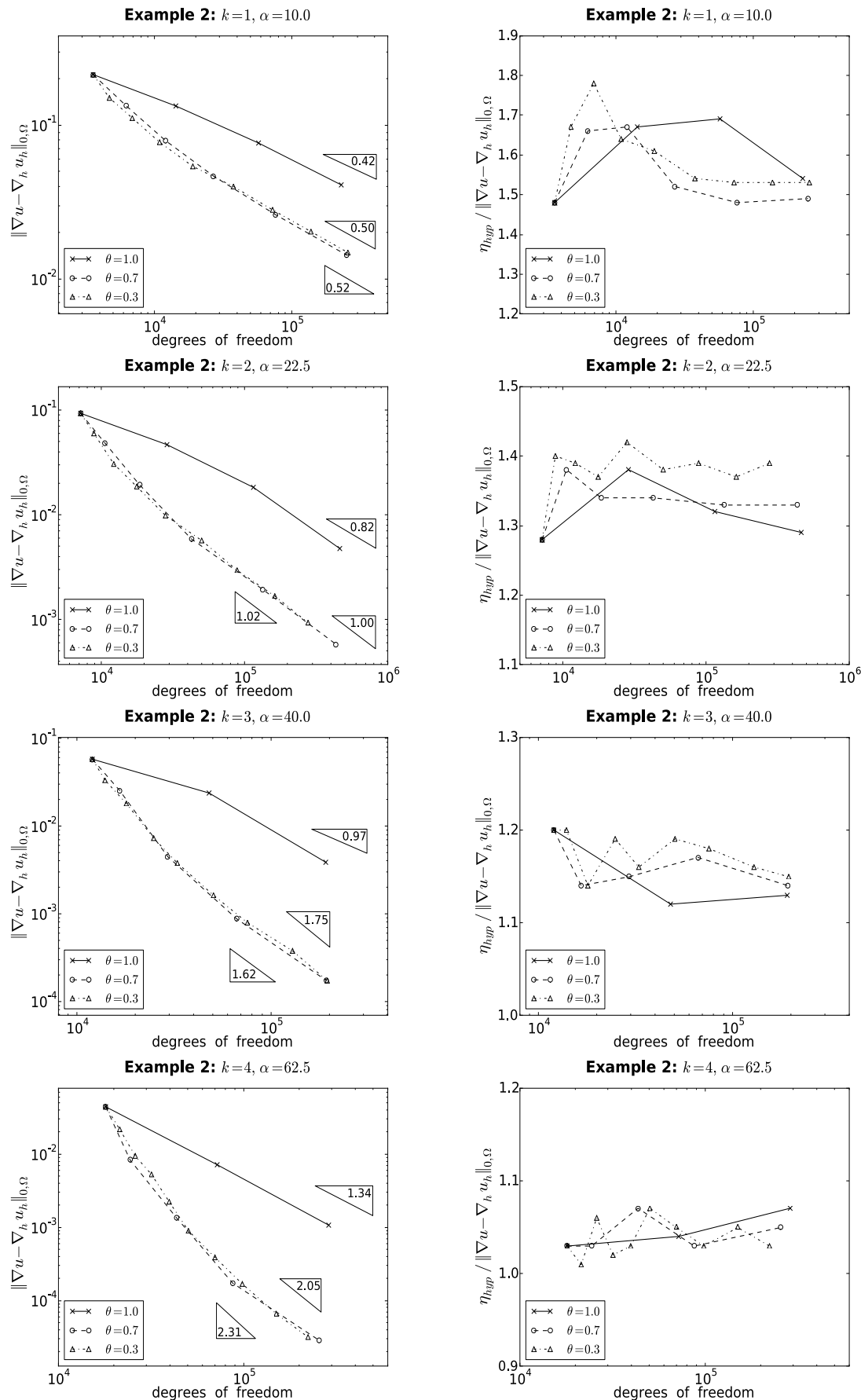

FIG. 8.4. Example 2: The discretization error $\left\|\operatorname{grad} u-\operatorname{grad}_{\mathrm{h}} u_{h}\right\|_{0, \Omega}$ as a function of the degrees of freedom (DOF) on a logarithmic scale for various $\theta$ in the Dörfler marking (left) and the associated effectivity indices (right). 
9. Concluding remarks. The design of the a posteriori error bound is the same for all discontinuous Galerkin methods. There is no generic constant, if data oscillations are excluded, and the proof of the reliability is much easier than that for residual-based estimators. In essence, it is focused on the terms which measure the nonconformity.

The proof of the efficiency is very similar to the analysis of residual-based error estimates, but there is one term less. The typical term

$$
h\left\|\Delta u_{h}+f\right\|_{0, \Omega}
$$

that models the negative norm $\left\|\Delta u_{h}+f\right\|_{-1}$ is not present, since implicitly a left inverse of the divergence operator is involved. The left inverse is constructed by a local procedure.

We recall that the analysis is based on the mixed formulation in [2], and it is known (see [5]) that the efficiency of the estimator is related to the quality of the mixed finite element method; cf. the comparison (7.6).

\section{REFERENCES}

[1] M. Ainsworth, A posteriori error estimation for lowest order Raviart Thomas mixed finite elements. SIAM J. Sci. Comput. 30, 189-204, 2008.

[2] D. Arnold, F. Brezzi, B. Cockburn, and D. Marini, Unified analysis of discontinuous Galerkin methods for elliptic problems. SIAM J. Numer. Anal. 39, 1749-1779, 2002.

[3] A. Bonito and R. H. Nochetto, Quasi-optimal convergence rate of an adaptive Discontinuous Galerkin method. SIAM J. Numer. Anal. 48, 734-771, 2010.

[4] D. Braess, Finite Elements: Theory, Fast Solvers and Applications in Solid Mechanics. 3rd edition. Cambridge University Press 2007.

[5] D. Braess, An a posteriori error estimate and a comparison theorem for the nonconforming $P_{1}$ element. Calcolo 46, 149-156, 2009.

[6] D. Braess, V. Pillwein, and J. Schöberl, Equilibrated residual error estimates are p-robust. Comp. Meth. Applied Mech. Engrg. 198, 1189-1197, 2009.

[7] D. Braess and J. Schöberl, Equilibrated residual error estimator for edge elements. Math. Comp. 77, 651-672, 2008.

[8] F. Brezzi and M. Fortin, Mixed and Hybrid Finite Element Methods. Springer, Berlin - Heidelberg - New York, 1991.

[9] J.M. Cascon, Ch. Kreuzer, R.H. Nochetto, and K.G. Siebert, Quasi-optimal rate of convergence of adaptive finite element methods. SIAM J. Numer. Anal. 46, 2524-2550, 2008.

[10] A. Ern and M. Vohralík, Flux reconstruction and a posteriori error estimation for discontinuous Galerkin methods on general nonmatching grids. C. R. Acad. Sci. Paris Ser. I 347, 44414444, 2009.

[11] J. S. Hesthaven and T. Warburton, Nodal Discontinuous Galerkin Methods: Algorithms, Analysis, and Applications. Springer, Berlin-Heidelberg-New York, 2008.

[12] R.H.W. Hoppe, G. Kanschat, and T. Warburton, Convergence analysis of an adaptive interior penalty discontinuous Galerkin method. SIAM J. Numer. Anal. 47, 534-550, 2009.

[13] O. A. Karakashian and F. Pascal, A posteriori error estimates for a discontinuous Galerkin approximation of second-order elliptic problems. SIAM J. Numer. Anal. 41, 2374-2399, 2003.

[14] O. A. Karakashian and F. Pascal, Convergence of adaptive discontinuous Galerkin approximations of second-order elliptic problems. SIAM J. Numer. Anal. 45, 641-665, 2007.

[15] W. Prager and J.L. Synge, Approximations in elasticity based on the concept of function spaces. Quart. Appl. Math. 5, 241-269, 1947. 